Proyecciones Journal of Mathematics

Vol. 35, No 4, pp. 437-455, December 2016.

Universidad Católica del Norte

Antofagasta - Chile

\title{
Sum divisor cordial labeling for star and ladder related graphs
}

\author{
A. Lourdusamy \\ St. Xavier's College (autonomous), India \\ and \\ F. Patrick \\ St. Xavier's College (autonomous), India \\ Received: April 2016. Accepted: October 2016
}

\begin{abstract}
$A$ sum divisor cordial labeling of a graph $G$ with vertex set $V$ is a bijection $f$ from $V$ to $\{1,2, \cdots,|V(G)|\}$ such that an edge uv is assigned the label 1 if 2 divides $f(u)+f(v)$ and 0 otherwise; and the number of edges labeled with 0 and the number of edges labeled with 1 differ by at most 1. A graph with a sum divisor cordial labeling is called a sum divisor cordial graph. In this paper, we prove that $D_{2}\left(K_{1, n}\right)$, $\left.S^{\prime}\left(K_{1, n}\right), D_{2}\left(B_{n, n}\right), D S\left(B_{n, n}\right), S^{\prime}\left(B_{n, n}\right), S\left(B_{n, n}\right),<K_{1, n}^{(1)} \Delta K_{1, n}^{(2)}\right\rangle$, $S\left(L_{n}\right), L_{n} \odot K_{1}, S L_{n}, T L_{n}, T L_{n} \odot K_{1}$ and $C H_{n}$ are sum divisor cordial graphs.
\end{abstract}

AMS Subject Classification 2010 : 05C78.

Keywords : Divisor cordial, sum divisor cordial. 


\section{Introduction}

All graphs considered here are simple, finite, connected and undirected. For all other standard terminology and notations we follow Harary [2]. A labeling of a graph is a map that carries the graph elements to the set of numbers, usually to the set of non-negative or positive integers. If the domain is the set of vertices the labeling is called vertex labeling. If the domain is the set of edges then the labeling is called edge labeling. If the labels are assigned to both vertices and edges then the labeling is called total labeling. For all detailed survey of graph labeling we refer Gallian [1]. A. Lourdusamy and F. Patrick introduced the concept of sum divisor cordial labeling in [3]. In [3, 4], sum divisor cordial labeling behaviour of several graphs like path, complete bipartite graph, bistar and some standard graphs have been investigated. In this paper, we investigate the sum divisor cordial labeling behavior of $D_{2}\left(K_{1, n}\right), S^{\prime}\left(K_{1, n}\right), D_{2}\left(B_{n, n}\right), D S\left(B_{n, n}\right), S^{\prime}\left(B_{n, n}\right)$, $S\left(B_{n, n}\right),<K_{1, n}^{(1)} \Delta K_{1, n}^{(2)}>, S\left(L_{n}\right), L_{n} \odot K_{1}, S L_{n}, T L_{n}, T L_{n} \odot K_{1}$ and $C H_{n}$.

Definition 1.1. Let $G=(V(G), E(G))$ be a simple graph and $f: V(G) \rightarrow$ $\{1,2, \cdots,|V(G)|\}$ be a bijection. For each edge uv, assign the label 1 if $2 \mid(f(u)+f(v))$ and the label 0 otherwise. The function $f$ is called a sum divisor cordial labeling if $\left|e_{f}(0)-e_{f}(1)\right| \leq 1$. A graph which admits a sum divisor cordial labeling is called a sum divisor cordial graph.

Definition 1.2. [9] For every vertex $v \in V(G)$, the open neighbourhood set $N(v)$ is the set of all vertices adjacent to $v$ in $G$.

Definition 1.3. [8] For a graph $G$ the splitting graph $S^{\prime}(G)$ of a graph $G$ is obtained by adding a new vertex $v^{\prime}$ corresponding to each vertex $v$ of $G$ such that $N(v)=N\left(v^{\prime}\right)$.

Definition 1.4. [8] The shadow graph $D_{2}(G)$ of a connected graph $G$ is obtained by taking two copies of $G$, say $G^{\prime}$ and $G^{\prime \prime}$. Join each vertex $u^{\prime}$ in $G^{\prime}$ to the neighbours of corresponding vertex $u^{\prime \prime}$ in $G^{\prime \prime}$.

Definition 1.5. [8] Duplication of a vertex $v_{k}$ by a new edge $e=v_{k}^{\prime} v_{k}^{\prime \prime}$ in a graph $G$ produces a new graph $G^{\prime}$ such that $N\left(v_{k}^{\prime}\right) \cap N\left(v_{k}^{\prime \prime}\right)=v_{k}$.

Definition 1.6. [8] Let $G$ be the a graph with $V=S_{1} \cup S_{2} \cup S_{3} \cup \cdots S_{i} \cup T$ where each $S_{i}$ is a set of vertices having at least two vertices of the same degree and $T=V(G) \backslash \cup_{i=1}^{t} S_{i}$. The degree splitting graph of $G$ denoted by $D S(G)$ is obtained from $G$ by adding vertices $w_{1}, w_{2}, \ldots, w_{t}$ and joining to each vertex of $S_{i}$ for $1 \leq i \leq t$. 
Definition 1.7. [6] The subdivision graph $S(G)$ is obtained from $G$ by subdividing each edge of $G$ with a vertex.

Definition 1.8. [7] Consider two copies of graph $G$ (wheel, star, fan and friendship) namely $G_{1}$ and $G_{2}$. Then the graph $G^{\prime}=<G_{1} \Delta G_{2}>$ is the graph obtained by joining the apex vertices of $G_{1}$ and $G_{2}$ by an edge as well as to a new vertex $v^{\prime}$.

Definition 1.9. [6] The slanting ladder $S L_{n}$ is a graph obtained from two paths $u_{1}, u_{2}, \cdots, u_{n}$ and $v_{1}, v_{2}, \cdots, v_{n}$ by joining each $u_{i}$ with $v_{i+1}$ for $(1 \leq$ $i \leq n-1)$.

Definition 1.10. [5] The triangular ladder $T L_{n}$ is a graph obtained from $L_{n}$ by adding the edges $u_{i} v_{i+1}, 1 \leq i \leq n-1$, where $u_{i}$ and $v_{i}, 1 \leq i \leq n$ are the vertices of $L_{n}$ such that $u_{1}, u_{2}, \cdots, u_{n}$ and $v_{1}, v_{2}, \cdots, v_{n}$ are two paths of length $n$ in the graph $L_{n}$.

Definition 1.11. [6] The corona $G_{1} \odot G_{2}$ of two graphs $G_{1}\left(p_{1}, q_{1}\right)$ and $G_{2}\left(p_{2}, q_{2}\right)$ is defined as the graph obtained by taking one copy of $G_{1}$ and $p_{1}$ copies of $G_{2}$ and joining the $i^{\text {th }}$ vertex of $G_{1}$ with an edge to every vertex in the $i^{\text {th }}$ copy of $G_{2}$.

Definition 1.12. [7] The closed helm $\mathrm{CH}_{n}$ is the graph obtained from helm $H_{n}$ by joining each pendant vertex to form a cycle.

\section{Main results}

Theorem 2.1. The graph $S^{\prime}\left(K_{1, n}\right)$ is sum divisor cordial graph.

Proof. Let $v_{1}, v_{2}, \cdots, v_{n}$ be the pendant vertices and $v$ be the apex vertex of $K_{1, n}$ and $u, u_{1}, u_{2}, \cdots, u_{n}$ are added vertices corresponding to $v, v_{1}, v_{2}, \cdots, v_{n}$ to obtain $S^{\prime}\left(K_{1, n}\right)$. Let $G=S^{\prime}\left(K_{1, n}\right)$. Then, $G$ is of order $2 n+2$ and size $3 n$. Define $f: V(G) \rightarrow\{1,2, \cdots, 2 n+2\}$ as follows:

$2 i+2$ if $i$ is even

$$
\begin{aligned}
& f(u)=1 \\
& f(v)=2 ; \\
& f\left(v_{i}\right)=\{2 i+1 \text { if } i \text { is odd }
\end{aligned}
$$

$2 i+1$ if $i$ is even

$$
f\left(u_{i}\right)=\{2 i+2 \text { if } i \text { is odd }
$$


Then, the induced edge labels are

$$
f^{*}\left(v u_{i}\right)=\{1 \text { if } i \text { is odd }
$$

Oif $i$ is even

lif $i$ is even

$$
f^{*}\left(v v_{i}\right)=\{0 \text { if } i \text { is odd }
$$

Oif $i$ is even

$$
f^{*}\left(u v_{i}\right)=\{1 \text { if } i \text { is odd }
$$

We observe that, $e_{f}(0)=\left\lfloor\frac{3 n}{2}\right\rfloor$ and $e_{f}(1)=\left\lceil\frac{3 n}{2}\right\rceil$.

Thus, $\left|e_{f}(1)-e_{f}(0)\right| \leq 1$.

Hence, $S^{\prime}\left(K_{1, n}\right)$ is sum divisor cordial graph.

Example 2.2. A sum divisor cordial labeling of $S^{\prime}\left(K_{1,5}\right)$ is shown in Figure 2.1

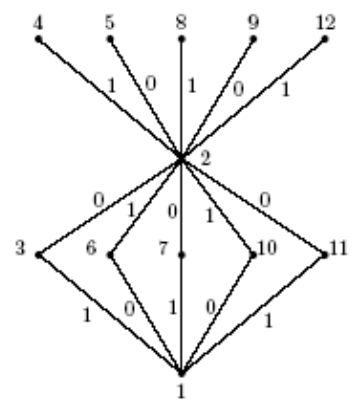

Figure 2.1

Theorem 2.3. The graph $D_{2}\left(K_{1, n}\right)$ is sum divisor cordial graph.

Proof. Let $u, u_{1}, u_{2}, \cdots, u_{n}$ and $v, v_{1}, v_{2}, \cdots, v_{n}$ be the vertices of two copies of $K_{1, n}$. Let $G=D_{2}\left(K_{1, n}\right)$. Then

$V(G)=\left\{u, v, u_{1}, u_{2}, \cdots, u_{n}, v_{1}, v_{2}, \cdots, v_{n}\right\}$ and $E(G)=\left\{u u_{i}, v v_{i}, u v_{i}, v u_{i}\right.$ : $1 \leq i \leq n\}$. Also, $G$ is of order $2 n+2$ and size $4 n$. Define $f: V(G) \rightarrow$ $\{1,2, \cdots, 2 n+2\}$ as follows:

$$
\begin{aligned}
& f(u)=1 ; \\
& f(v)=2 ;
\end{aligned}
$$


$2 i+2$ if $i$ is even

$$
f\left(u_{i}\right)=\{2 i+1 \text { if } i \text { is odd }
$$

$2 i+1$ if $i$ is even

$$
f\left(v_{i}\right)=\{2 i+2 \text { if } i \text { is odd }
$$

Then, the induced edge labels are

Oif $i$ is even

$$
f^{*}\left(u u_{i}\right)=\{1 \text { if } i \text { is odd }
$$

lif $i$ is even

$$
f^{*}\left(v u_{i}\right)=\{0 \text { if } i \text { is odd }
$$

Oif $i$ is even

$$
f^{*}\left(v v_{i}\right)=\{1 \text { if } i \text { is odd }
$$

lif $i$ is even

$$
f^{*}\left(u v_{i}\right)=\{0 \text { if } i \text { is odd }
$$

We observe that, $e_{f}(0)=2 n$ and $e_{f}(1)=2 n$.

Thus, $\left|e_{f}(1)-e_{f}(0)\right| \leq 1$.

Hence, $D_{2}\left(K_{1, n}\right)$ is sum divisor cordial graph.

Example 2.4. A sum divisor cordial labeling of $D_{2}\left(K_{1,4}\right)$ is shown in Figure 2.2

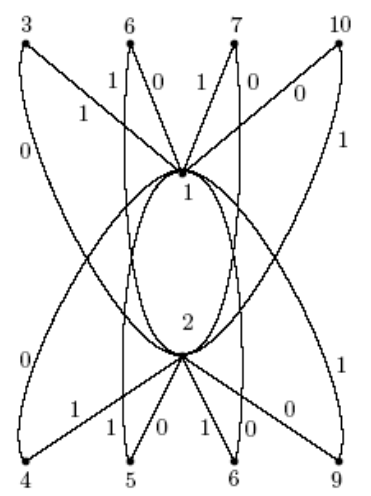

Figure 2.2

Theorem 2.5. The graph $S^{\prime}\left(B_{n, n}\right)$ is sum divisor cordial graph. 
Proof. Let $u, v, u_{1}, u_{2}, \cdots, u_{n}, v_{1}, v_{2}, \cdots, v_{n}$ be the vertices of $B_{n, n}$. Let $u^{\prime}, v^{\prime}, u_{i}^{\prime}, v_{i}^{\prime}$ are added vertices corresponding to $u, v, u_{i}, v_{i}$ to obtain $S^{\prime}\left(B_{n, n}\right)$. Let $G=S^{\prime}\left(B_{n, n}\right)$ Then, $G$ is of order $4 n+4$ and size $6 n+3$. Define $f: V(G) \rightarrow\{1,2, \cdots, 4 n+4\}$ as follows:

$$
\begin{aligned}
& f(u)=1 ; \\
& f(v)=3 ; \\
& f\left(u^{\prime}\right)=2 ; \\
& f\left(v^{\prime}\right)=4 ; \\
& f\left(u_{i}\right)=4 i+2 ; 1 \leq i \leq n \\
& f\left(u_{i}^{\prime}\right)=4 i+1 ; 1 \leq i \leq n \\
& f\left(v_{i}\right)=4 i+3 ; 1 \leq i \leq n \\
& f\left(v_{i}^{\prime}\right)=4 i+4 ; 1 \leq i \leq n
\end{aligned}
$$

Then, the induced edge labels are

$$
\begin{aligned}
& f^{*}(u v)=1 ; \\
& f^{*}\left(u v^{\prime}\right)=0 ; \\
& f^{*}\left(v u^{\prime}\right)=0 ; \\
& f^{*}\left(u^{\prime} u_{i}\right)=1 ; 1 \leq i \leq n \\
& f^{*}\left(u u_{i}\right)=0 ; 1 \leq i \leq n \\
& f^{*}\left(u u_{i}^{\prime}\right)=1 ; 1 \leq i \leq n \\
& f^{*}\left(v^{\prime} v_{i}\right)=0 ; 1 \leq i \leq n \\
& f^{*}\left(v v_{i}\right)=1 ; 1 \leq i \leq n \\
& f^{*}\left(v v_{i}^{\prime}\right)=0 ; 1 \leq i \leq n
\end{aligned}
$$

We observe that, $e_{f}(0)=3 n+2$ and $e_{f}(1)=3 n+1$.

Thus, $\left|e_{f}(1)-e_{f}(0)\right| \leq 1$.

Hence, $S^{\prime}\left(B_{n, n}\right)$ is sum divisor cordial graph.

Example 2.6. A sum divisor cordial labeling of $S^{\prime}\left(B_{5,5}\right)$ is shown in Figure 2.3 


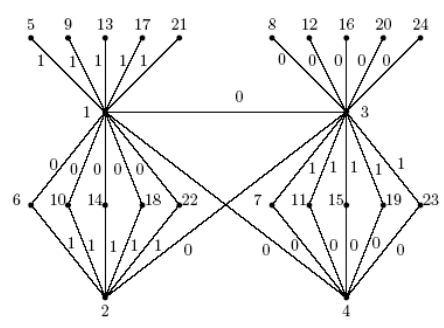

Figure 2.3

Theorem 2.7. The graph $D_{2}\left(B_{n, n}\right)$ is sum divisor cordial graph.

Proof. Let $u, v, u_{1}, u_{2}, \cdots, u_{n}, v_{1}, v_{2}, \cdots, v_{n}$ be the vertices of $B_{n, n}$. Let $G=D_{2}\left(B_{n, n}\right)$. Then $V(G)=\left\{u, v, u^{\prime}, v^{\prime}\right\} \bigcup\left\{u_{i}, v_{i}, u_{i}^{\prime}, v_{i}^{\prime}: 1 \leq i \leq n\right\}$ and $E(G)=\left\{u v, u^{\prime} v, u v^{\prime}, u^{\prime} v^{\prime}\right\} \bigcup\left\{u u_{i}, u u_{i}^{\prime}, u^{\prime} u_{i}, u^{\prime} u_{i}^{\prime}, v v_{i}, v v_{i}^{\prime}, v^{\prime} v_{i}, v^{\prime} v_{i}^{\prime}: 1 \leq i \leq\right.$ $n\}$. Also, $G$ is of order $4 n+4$ and size $8 n+4$. Define $f: V(G) \rightarrow$ $\{1,2, \cdots, 4 n+4\}$ as follows:

$$
\begin{aligned}
& f(u)=1 ; \\
& f(v)=3 ; \\
& f\left(u^{\prime}\right)=2 ; \\
& f\left(v^{\prime}\right)=4 \\
& f\left(u_{i}\right)=4 i+1 ; 1 \leq i \leq n \\
& f\left(u_{i}^{\prime}\right)=4 i+2 ; 1 \leq i \leq n \\
& f\left(v_{i}\right)=4 i+4 ; 1 \leq i \leq n \\
& f\left(v_{i}^{\prime}\right)=4 i+3 ; 1 \leq i \leq n
\end{aligned}
$$

Then, the induced edge labels are

$$
\begin{aligned}
& f^{*}(u v)=1 ; \\
& f^{*}\left(u v^{\prime}\right)=0 ; \\
& f^{*}\left(v u^{\prime}\right)=0 ; \\
& f^{*}\left(v^{\prime} u^{\prime}\right)=1 ; \\
& f^{*}\left(u u_{i}\right)=1 ; 1 \leq i \leq n \\
& f^{*}\left(u^{\prime} u_{i}\right)=0 ; 1 \leq i \leq n \\
& f^{*}\left(u^{\prime} u_{i}^{\prime}\right)=1 ; 1 \leq i \leq n
\end{aligned}
$$




$$
\begin{aligned}
& f^{*}\left(u u_{i}^{\prime}\right)=0 ; 1 \leq i \leq n \\
& f^{*}\left(v v_{i}\right)=0 ; 1 \leq i \leq n \\
& f^{*}\left(v^{\prime} v_{i}\right)=1 ; 1 \leq i \leq n \\
& f^{*}\left(v^{\prime} v_{i}^{\prime}\right)=0 ; 1 \leq i \leq n \\
& f^{*}\left(v v_{i}^{\prime}\right)=1 ; 1 \leq i \leq n
\end{aligned}
$$

We observe that, $e_{f}(0)=4 n+2$ and $e_{f}(1)=4 n+2$.

Thus, $\left|e_{f}(1)-e_{f}(0)\right| \leq 1$.

Hence, $D_{2}\left(B_{n, n}\right)$ is sum divisor cordial graph.

Example 2.8. A sum divisor cordial labeling of $D_{2}\left(B_{4,4}\right)$ is shown in Figure 2.4

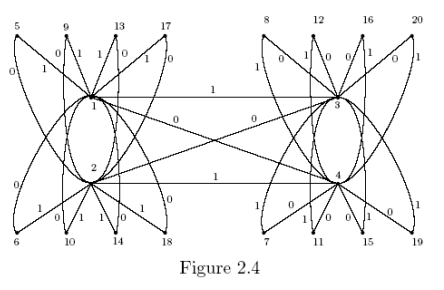

Theorem 2.9. The graph $D S\left(B_{n, n}\right)$ is sum divisor cordial graph.

Proof. $\quad$ Let $G=D S\left(B_{n, n}\right)$. Let $V(G)=\left\{u, v, w_{1}, w_{2}\right\} \bigcup\left\{u_{i}, v_{i}: 1 \leq i \leq\right.$ $n\}$ and $E(G)=\left\{u v, u w_{2}, v w_{2}\right\} \bigcup\left\{u u_{i}, v v_{i}, u_{i} w_{1}, v_{i} w_{1}: 1 \leq i \leq n\right\}$. Then, $G$ is of order $2 n+4$ and size $4 n+3$. Define $f: V(G) \rightarrow\{1,2, \cdots, 2 n+4\}$ as follows:

$$
\begin{aligned}
& f(u)=4 ; \\
& f(v)=2 ; \\
& f\left(w_{1}\right)=1 ; \\
& f\left(w_{2}\right)=3 ; \\
& f\left(u_{i}\right)=2 i+4 ; 1 \leq i \leq n \\
& f\left(v_{i}\right)=2 i+3 ; 1 \leq i \leq n
\end{aligned}
$$

Then, the induced edge labels are 


$$
\begin{aligned}
& f^{*}(u v)=1 ; \\
& f^{*}\left(u w_{2}\right)=0 ; \\
& f^{*}\left(v w_{2}\right)=0 ; \\
& f^{*}\left(u u_{i}\right)=1 ; 1 \leq i \leq n \\
& f^{*}\left(u_{i} w_{1}\right)=0 ; 1 \leq i \leq n \\
& f^{*}\left(v v_{i}\right)=0 ; 1 \leq i \leq n \\
& f^{*}\left(v_{i} w_{1}\right)=1 ; 1 \leq i \leq n
\end{aligned}
$$

We observe that, $e_{f}(0)=2 n+2$ and $e_{f}(1)=2 n+1$.

Thus, $\left|e_{f}(1)-e_{f}(0)\right| \leq 1$.

Hence, $D S\left(B_{n, n}\right)$ is sum divisor cordial graph.

Example 2.10. A sum divisor cordial labeling of $D S\left(B_{3,3}\right)$ is shown in Figure 2.5

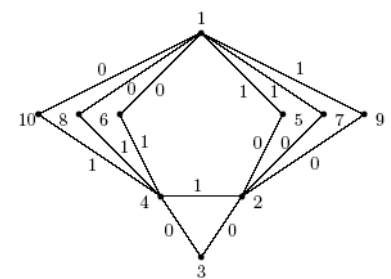

Figure 2.5

Theorem 2.11. The graph $S\left(B_{n, n}\right)$ is sum divisor cordial graph.

Proof. $\quad$ Let $G=S\left(B_{n, n}\right)$. Let $V(G)=\{u, v, w\} \bigcup\left\{u_{i}, v_{i}, u_{i}^{\prime}, v_{i}^{\prime}: 1 \leq i \leq\right.$ $n\}$ and $E(G)=\{u w, w v\} \bigcup\left\{u u_{i}^{\prime}, u_{i}^{\prime} u_{i}, v v_{i}^{\prime}, v_{i}^{\prime} v_{i}: 1 \leq i \leq n\right\}$. Then, $G$ is of order $4 n+3$ and size $4 n+2$. Define $f: V(G) \rightarrow\{1,2, \cdots, 4 n+3\}$ as follows:

$$
\begin{aligned}
& f(u)=1 ; \\
& f(v)=2 ; \\
& f(w)=3 \\
& f\left(u_{i}\right)=4 i ; 1 \leq i \leq n \\
& f\left(u_{i}^{\prime}\right)=4 i+1 ; 1 \leq i \leq n
\end{aligned}
$$




$$
\begin{aligned}
& f\left(v_{i}\right)=4 i+3 ; 1 \leq i \leq n \\
& f\left(v_{i}^{\prime}\right)=4 i+2 ; 1 \leq i \leq n
\end{aligned}
$$

Then, the induced edge labels are

$$
\begin{aligned}
& f^{*}(u w)=1 ; \\
& f^{*}(w v)=0 ; \\
& f^{*}\left(u u_{i}^{\prime}\right)=1 ; 1 \leq i \leq n \\
& f^{*}\left(u_{i}^{\prime} u_{i}\right)=0 ; 1 \leq i \leq n \\
& f^{*}\left(v v_{i}^{\prime}\right)=1 ; 1 \leq i \leq n \\
& f^{*}\left(v_{i}^{\prime} v_{i}\right)=0 ; 1 \leq i \leq n
\end{aligned}
$$

We observe that, $e_{f}(0)=2 n+1$ and $e_{f}(1)=2 n+1$.

Thus, $\left|e_{f}(1)-e_{f}(0)\right| \leq 1$.

Hence, $S\left(B_{n, n}\right)$ is sum divisor cordial graph.

Example 2.12. A sum divisor cordial labeling of $S\left(B_{3,3}\right)$ is shown in Figure 2.6

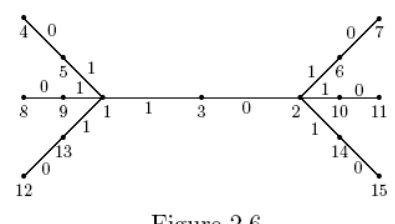

Figure 2.6

Theorem 2.13. The graph $<K_{1, n}^{(1)} \Delta K_{1, n}^{(2)}>$ is sum divisor cordial graph.

Proof. Let $v_{1}^{(1)}, v_{2}^{(1)}, \cdots, v_{n}^{(1)}$ be the pendant vertices of $K_{1, n}^{(1)}$ and $v_{1}^{(2)}, v_{2}^{(2)}, \cdots, v_{n}^{(2)}$ be the pendant vertices of $K_{1, n}^{(2)}$. Let $u$ and $v$ be the apex vertices of $K_{1, n}^{(1)}$ and $K_{1, n}^{(2)}$ respectively and $u, v$ are adjacent to a new common vertex $x$. Let $G=<K_{1, n}^{(1)} \Delta K_{1, n}^{(2)}>$. Then, $G$ is of order $2 n+3$ and size $2 n+3$. Define $f: V(G) \rightarrow\{1,2, \cdots, 2 n+3\}$ as follows:

$$
f(x)=2 \text {; }
$$


Then, the induced edge labels are

$$
\begin{aligned}
& f(u)=1 ; \\
& f(v)=3 ; \\
& f\left(u_{2 i-1}\right)=4 i ; 1 \leq i \leq\left\lceil\frac{n}{2}\right\rceil \\
& f\left(u_{2 i}\right)=4 i+3 ; 1 \leq i \leq\left\lfloor\frac{n}{2}\right\rfloor \\
& f\left(v_{2 i-1}\right)=4 i+1 ; 1 \leq i \leq\left\lceil\frac{n}{2}\right\rceil \\
& f\left(v_{2 i}\right)=4 i+2 ; 1 \leq i \leq\left\lfloor\frac{n}{2}\right\rfloor
\end{aligned}
$$

$$
\begin{aligned}
& f^{*}(u x)=0 ; \\
& f^{*}(v x)=0 \\
& f^{*}(u v)=1 ; \\
& f^{*}\left(u u_{2 i-1}\right)=0 ; 1 \leq i \leq\left\lceil\frac{n}{2}\right\rceil \\
& f^{*}\left(u u_{2 i}\right)=1 ; 1 \leq i \leq\left\lfloor\frac{n}{2}\right\rfloor \\
& f^{*}\left(v v_{2 i-1}\right)=1 ; 1 \leq i \leq\left\lceil\frac{n}{2}\right\rceil \\
& f^{*}\left(v v_{2 i}\right)=0 ; 1 \leq i \leq\left\lfloor\frac{n}{2}\right\rfloor
\end{aligned}
$$

We observe that, $e_{f}(0)=n+2$ and $e_{f}(1)=n+1$.

Thus, $\left|e_{f}(1)-e_{f}(0)\right| \leq 1$.

Hence, $<K_{1, n}^{(1)} \Delta K_{1, n}^{(2)}>$ is sum divisor cordial graph.

Example 2.14. A sum divisor cordial labeling of $<K_{1,5}^{(1)} \Delta K_{1,5}^{(2)}>$ is shown in Figure 2.7

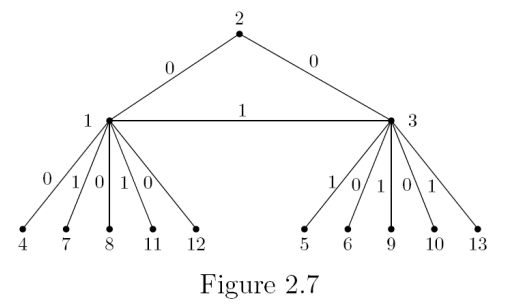

Theorem 2.15. The graph $L_{n} \odot K_{1}$ is sum divisor cordial graph.

Proof. Let $u_{1}, u_{2}, \cdots, u_{n}$ and $v_{1}, v_{2}, \cdots, v_{n}$ be the vertices of $L_{n}$. Let $x_{i}$ be the vertex which is attached to $u_{i}$ and $y_{i}$ be the vertex which is attached 
to $v_{i}$. Let $G=L_{n} \odot K_{1}$. Then, $G$ is of order $4 n$ and size $5 n-2$. Define $f: V(G) \rightarrow\{1,2, \cdots, 4 n\}$ as follows:

$$
\begin{aligned}
& f\left(u_{2 i-1}\right)=8 i-5 ; 1 \leq i \leq\left\lceil\frac{n}{2}\right\rceil \\
& f\left(u_{2 i}\right)=8 i-3 ; 1 \leq i \leq\left\lfloor\frac{n}{2}\right\rfloor \\
& f\left(x_{2 i-1}\right)=8 i-7 ; 1 \leq i \leq\left\lceil\frac{n}{2}\right\rceil \\
& f\left(x_{2 i}\right)=8 i-2 ; 1 \leq i \leq\left\lfloor\frac{n}{2}\right\rfloor \\
& f\left(v_{2 i-1}\right)=8 i-6 ; 1 \leq i \leq\left\lceil\frac{n}{2}\right\rceil \\
& f\left(v_{2 i}\right)=8 i-1 ; 1 \leq i \leq\left\lfloor\frac{n}{2}\right\rfloor \\
& f\left(y_{2 i-1}\right)=8 i-4 ; 1 \leq i \leq\left\lceil\frac{n}{2}\right\rceil \\
& f\left(y_{2 i}\right)=8 i ; 1 \leq i \leq\left\lfloor\frac{n}{2}\right\rfloor
\end{aligned}
$$

Then, the induced edge labels are

$$
\begin{aligned}
& f^{*}\left(u_{2 i-1} x_{2 i-1}\right)=1 ; 1 \leq i \leq\left\lceil\frac{n}{2}\right\rceil \\
& f^{*}\left(u_{2 i} x_{2 i}\right)=0 ; 1 \leq i \leq\left\lfloor\frac{n}{2}\right\rfloor \\
& f^{*}\left(u_{i} u_{i+1}\right)=1 ; 1 \leq i \leq n-1 \\
& f^{*}\left(v_{i} v_{i+1}\right)=0 ; 1 \leq i \leq n-1 \\
& f^{*}\left(u_{2 i-1} v_{2 i-1}\right)=0 ; 1 \leq i \leq\left\lceil\frac{n}{2}\right\rceil \\
& f^{*}\left(u_{2 i} v_{2 i}\right)=1 ; 1 \leq i \leq\left\lfloor\frac{n}{2}\right\rfloor \\
& f^{*}\left(v_{2 i-1} y_{2 i-1}\right)=1 ; 1 \leq i \leq\left\lceil\frac{n}{2}\right\rceil \\
& f^{*}\left(v_{2 i} y_{2 i}\right)=0 ; 1 \leq i \leq\left\lfloor\frac{n}{2}\right\rfloor
\end{aligned}
$$

We observe that, $e_{f}(0)=\left\lfloor\frac{5 n-2}{2}\right\rfloor$ and $e_{f}(1)=\left\lceil\frac{5 n-2}{2}\right\rceil$.

Thus, $\left|e_{f}(1)-e_{f}(0)\right| \leq 1$.

Hence, $L_{n} \odot K_{1}$ is sum divisor cordial graph.

Example 2.16. A sum divisor cordial labeling of $L_{6} \odot K_{1}$ is shown in Figure 2.8 


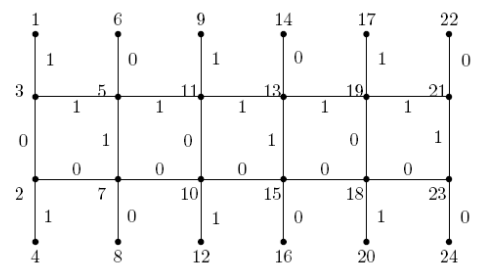

Figure 2.8

Theorem 2.17. The graph $S\left(L_{n}\right)$ is sum divisor cordial graph.

Proof. Let $u_{1}, u_{2}, \cdots, u_{n}$ and $v_{1}, v_{2}, \cdots, v_{n}$ be the vertices of $L_{n}$. Let $v_{i}^{\prime}$ be the newly added vertex between $v_{i}$ and $v_{i+1}$. Let $u_{i}^{\prime}$ be the newly added vertex between $u_{i}$ and $u_{i+1}$. Let $w_{i}$ be the newly added vertex between $v_{i}$ and $u_{i}$. Let $G=S\left(L_{n}\right)$. Then, $G$ is of order $5 n-2$ and size $6 n-4$. Define $f: V(G) \rightarrow\{1,2, \cdots, 5 n-2\}$ as follows:

$$
\begin{aligned}
& f\left(v_{1}\right)=1 ; \\
& f\left(v_{i+1}\right)=5 i ; 1 \leq i \leq n-1 \\
& f\left(u_{1}\right)=2 ; \\
& f\left(u_{i+1}\right)=5 i+3 ; 1 \leq i \leq n-1 \\
& f\left(w_{1}\right)=3 ; \\
& f\left(w_{i+1}\right)=5 i+2 ; 1 \leq i \leq n-1 \\
& f\left(v_{i}^{\prime}\right)=5 i-1 ; 1 \leq i \leq n-1 \\
& f\left(u_{i}^{\prime}\right)=5 i+1 ; 1 \leq i \leq n-1
\end{aligned}
$$

Then, the induced edge labels are

$$
\begin{aligned}
& f^{*}\left(v_{1} v_{1}^{\prime}\right)=0 ; \\
& f^{*}\left(v_{i} v_{i}^{\prime}\right)=1 ; 2 \leq i \leq n-1 \\
& f^{*}\left(v_{i}^{\prime} v_{i+1}\right)=0 ; 1 \leq i \leq n-1 \\
& f^{*}\left(u_{1} u_{1}^{\prime}\right)=1 ; \\
& f^{*}\left(u_{i} u_{i}^{\prime}\right)=0 ; 2 \leq i \leq n-1 \\
& f^{*}\left(u_{i}^{\prime} u_{i+1}\right)=1 ; 1 \leq i \leq n-1 \\
& f^{*}\left(v_{i} w_{i}\right)=1 ; 1 \leq i \leq n
\end{aligned}
$$




$$
f^{*}\left(u_{i} w_{i}\right)=0 ; 1 \leq i \leq n
$$

We observe that, $e_{f}(0)=3 n-2$ and $e_{f}(1)=3 n-2$.

Thus, $\left|e_{f}(1)-e_{f}(0)\right| \leq 1$.

Hence, $S\left(L_{n}\right)$ is sum divisor cordial graph.

Example 2.18. A sum divisor cordial labeling of $S\left(L_{5}\right)$ is shown in Figure 2.9

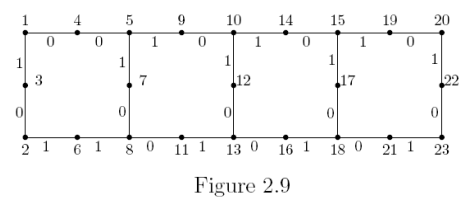

Theorem 2.19. The slanting ladder $S L_{n}$ is sum divisor cordial graph.

Proof. Let $G=S L_{n}$. Let $V(G)=\left\{u_{i}, v_{i}: 1 \leq i \leq n\right\}$ and $E(G)=$ $\left\{v_{i} v_{i+1}, u_{i} u_{i+1}, u_{i} v_{i+1}: 1 \leq i \leq n-1\right\}$. Then, $G$ is of order $2 n$ and size $3 n-3$. Define $f: V(G) \rightarrow\{1,2, \cdots, 2 n\}$ as follows:

$2 i-1$ if $i \equiv 2,3(\bmod 4)$

$$
f\left(u_{i}\right)=\{2 \text { iff } i \equiv 1,0(\bmod 4)
$$

$2 i$ if $i \equiv 2,3(\bmod 4)$

$$
f\left(v_{i}\right)=\{2 i-1 \text { if } i \equiv 1,0(\bmod 4)
$$

Then, the induced edge labels are

lif $i$ is even

$$
f^{*}\left(u_{i} u_{i+1}\right)=\{0 \text { if } i \text { is odd }
$$

lif $i$ is even

$$
f^{*}\left(v_{i} v_{i+1}\right)=\{0 \text { if } i \text { is odd }
$$

$$
f^{*}\left(u_{i} v_{i+1}\right)=\{1 \text { if } i \text { is odd }
$$

Oif $i$ is even

We observe that, $e_{f}(0)=\left\lceil\frac{3 n-3}{2}\right\rceil$ and $e_{f}(1)=\left\lfloor\frac{3 n-3}{2}\right\rfloor$. 
Thus, $\left|e_{f}(0)-e_{f}(1)\right| \leq 1$.

Hence, $S L_{n}$ is sum divisor cordial graph.

Example 2.20. A sum divisor cordial labeling of $S L_{5}$ is shown in Figure 2.10

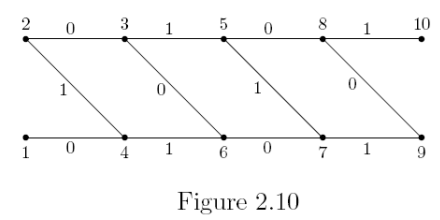

Theorem 2.21. The triangular ladder $T L_{n}$ is sum divisor cordial graph.

Proof. Let $G=T L_{n}$. Let $V(G)=\left\{u_{i}, v_{i}: 1 \leq i \leq n\right\}$ and $E(G)=$ $\left\{u_{i} u_{i+1}, v_{i} v_{i+1}, u_{i} v_{i+1}: 1 \leq i \leq n-1\right\} \bigcup\left\{u_{i} v_{i}: 1 \leq i \leq n\right\}$. Then, $G$ is of order $2 n$ and size $4 n-3$. Define $f: V(G) \rightarrow\{1,2, \cdots, 2 n\}$ as follows:

$$
\begin{aligned}
& f\left(u_{i}\right)=2 i-1 ; 1 \leq i \leq n \\
& f\left(v_{i}\right)=2 i ; 1 \leq i \leq n
\end{aligned}
$$

Then, the induced edge labels are

$$
\begin{aligned}
& f^{*}\left(u_{i} u_{i+1}\right)=1 ; 1 \leq i \leq n-1 \\
& f^{*}\left(u_{i} v_{i}\right)=0 ; 1 \leq i \leq n \\
& f^{*}\left(v_{i} v_{i+1}\right)=1 ; 1 \leq i \leq n-1 \\
& f^{*}\left(u_{i} v_{i+1}\right)=0 ; 1 \leq i \leq n-1
\end{aligned}
$$

We observe that, $e_{f}(0)=2 n-1$ and $e_{f}(1)=2 n-2$.

Thus, $\left|e_{f}(0)-e_{f}(1)\right| \leq 1$.

Hence, $T L_{n}$ is sum divisor cordial graph.

Example 2.22. A sum divisor cordial labeling of $T L_{5}$ is shown in Figure 2.11 


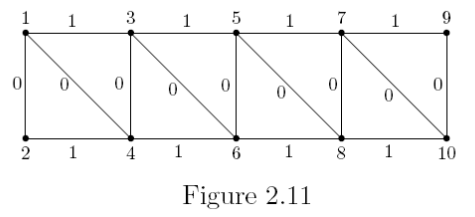

Theorem 2.23. The graph $T L_{n} \odot K_{1}$ is sum divisor cordial graph.

Proof. Let $u_{1}, u_{2}, \cdots, u_{n}$ and $v_{1}, v_{2}, \cdots, v_{n}$ be the vertices of $T L_{n}$. Let $G=T L_{n} \odot K_{1}$. Then $V(G)=\left\{u_{i}, v_{i}, x_{i}, y_{i}: 1 \leq i \leq n\right\}$ and $E(G)=$ $\left\{u_{i} u_{i+1}, v_{i} v_{i+1}, u_{i} v_{i+1}: 1 \leq i \leq n-1\right\} \bigcup\left\{u_{i} x_{i}, v_{i} y_{i}, u_{i} v_{i}: 1 \leq i \leq n\right\}$. Then, $G$ is of order $4 n$ and size $6 n-3$. Define $f: V(G) \rightarrow\{1,2, \cdots, 4 n\}$ as follows:

$4 i-3$ if $i$ is even

$$
f\left(u_{i}\right)=\{4 i-1 \text { if } i \text { is odd }
$$

$4 i-2$ if $i$ is even

$$
f\left(x_{i}\right)=\{4 i-3 \text { if } i \text { is odd }
$$

$$
f\left(v_{i}\right)=\{4 i-2 \text { if } i \text { is odd }
$$

$4 i-1$ if $i$ is even

$$
f\left(y_{i}\right)=4 i ; 1 \leq i \leq n
$$

Then, the induced edge labels are

$$
f^{*}\left(u_{i} x_{i}\right)=\{1 \text { if } i \text { is odd }
$$

0 if $i$ is even

$$
f^{*}\left(v_{i} y_{i}\right)=\{1 \text { if } i \text { is odd }
$$

0 if $i$ is even

$$
f^{*}\left(u_{i} v_{i}\right)=\{0 \text { if } i \text { is odd }
$$

1 if $i$ is even

$$
f^{*}\left(u_{i} v_{i+1}\right)=\{1 \text { if } i \text { is odd }
$$

0 if $i$ is even 


$$
\begin{aligned}
& f^{*}\left(u_{i} u_{i+1}\right)=1 ; 1 \leq i \leq n-1 \\
& f^{*}\left(v_{i} v_{i+1}\right)=0 ; 1 \leq i \leq n-1
\end{aligned}
$$

We observe that, $e_{f}(0)=3 n-2$ and $e_{f}(1)=3 n-1$.

Thus, $\left|e_{f}(1)-e_{f}(0)\right| \leq 1$.

Hence, $T L_{n} \odot K_{1}$ is sum divisor cordial graph.

Example 2.24. A sum divisor cordial labeling of $T L_{6} \odot K_{1}$ is shown in Figure 2.12

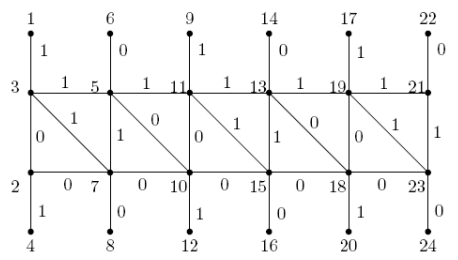

Figure 2.12

Theorem 2.25. The closed helm graph $\mathrm{CH}_{n}$ is sum divisor cordial graph.

Proof. Let $G=C H_{n}$. Let $V(G)=\left\{v, v_{1}, v_{2}, \cdots, v_{n}, u_{1}, u_{2}, \cdots, u_{n}\right\}$ and $E(G)=\left\{v v_{i}, u_{i} v_{i}: 1 \leq i \leq n\right\} \bigcup\left\{v_{i} v_{i+1}, u_{i} u_{i+1}: 1 \leq i \leq n-\right.$ $1\} \bigcup\left\{v_{1} v_{n}, u_{1} u_{n}\right\}$. Then, $G$ is of order $2 n+1$ and size $4 n$. Define $f$ : $V(G) \rightarrow\{1,2, \cdots, 2 n+1\}$ as follows:

$$
\begin{aligned}
& f(v)=1 ; \\
& f\left(v_{i}\right)=2 i ; 1 \leq i \leq n \\
& f\left(u_{i}\right)=2 i+1 ; 1 \leq i \leq n
\end{aligned}
$$

Then, the induced edge labels are

$$
\begin{aligned}
& f^{*}\left(v v_{i}\right)=0 ; 1 \leq i \leq n \\
& f^{*}\left(v_{i} v_{i+1}\right)=1 ; 1 \leq i \leq n-1 \\
& f^{*}\left(v_{1} v_{n}\right)=1 ; \\
& f^{*}\left(u_{i} v_{i}\right)=0 ; 1 \leq i \leq n \\
& f^{*}\left(u_{i} u_{i+1}\right)=1 ; 1 \leq i \leq n-1 \\
& f^{*}\left(u_{1} u_{n}\right)=1 ;
\end{aligned}
$$


We observe that, $e_{f}(0)=2 n$ and $e_{f}(1)=2 n$.

Thus, $\left|e_{f}(1)-e_{f}(0)\right| \leq 1$.

Hence, $C H_{n}$ is sum divisor cordial graph.

Example 2.26. A sum divisor cordial labeling of $\mathrm{CH}_{4}$ is shown in Figure 2.13

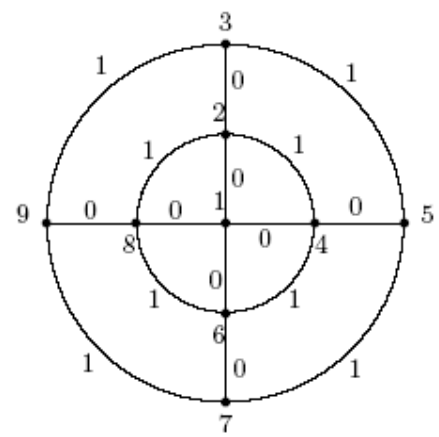

Figure 2.13

\section{3. conclusion}

It is very interesting and challenging as well as to investigate graph families which admit sum divisor cordial labeling. Here we have proved $D_{2}\left(K_{1, n}\right)$, $S^{\prime}\left(K_{1, n}\right), D_{2}\left(B_{n, n}\right), D S\left(B_{n, n}\right), S^{\prime}\left(B_{n, n}\right), S\left(B_{n, n}\right),<K_{1, n}^{(1)} \Delta K_{1, n}^{(2)}>, S\left(L_{n}\right)$, $L_{n} \odot K_{1}, S L_{n}, T L_{n}, T L_{n} \odot K_{1}$ and $C H_{n}$ are sum divisor cordial graphs.

\section{References}

[1] J. A. Gallian, A Dyamic Survey of Graph Labeling, The Electronic J. Combin., 18 (2015) \# DS6.

[2] F. Harary, Graph Theory, Addison-wesley, Reading, Mass, (1972).

[3] A. Lourdusamy and F. Patrick, Sum Divisor Cordial Graphs, Proyecciones Journal of Mathematics, 35(1), pp. 115-132, (2016). 
[4] A. Lourdusamy and F. Patrick, Sum Divisor Cordial Labeling for Path and Cycle Related Graphs (submitted for publication)

[5] S. S. Sandhya, S. Somasundaram and S. Anusa Some New Results on Root Square Mean Labeling, International Journal of Mathematical Archive, 5(12), pp. 130-135, (2014).

[6] M. Seenivasan Some New Labeling Concepts, PhD thesis, Manonmaniam Sundaranar University, India, (2013).

[7] S. K. Vaidya and C. M. Barasara, Product Cordial Graphs in the Context of Some Graph Operations, International Journal of Mathematics and Scientific Computing, 1(2), pp. 122-130, (2011).

[8] S. K. Vaidya and N. H. Shah, Some Star and Bistar Related Cordial Graphs, Annals of Pure and Applied Mathematics, 3(1), pp. 67-77, (2013).

[9] S. K. Vaidya and N. J. Kothari, Line Gracefulness of Some Path Related Graphs, International Journal of Mathematics and Scientific Computing, 4(1), pp. 15-18, (2014).

\author{
A. Lourdusamy \\ Department of Mathematics, \\ St. Xavier's College (autonomous), \\ Palayamkottai-627002, \\ Tamilnadu, \\ India \\ e-mail : lourdusamy15@gmail.com \\ and \\ F. Patrick \\ Department of Mathematics, \\ St. Xavier's College (autonomous), \\ Palayamkottai-627002, \\ Tamilnadu, \\ India \\ e-mail : patrick881990@gmail.com
}

